
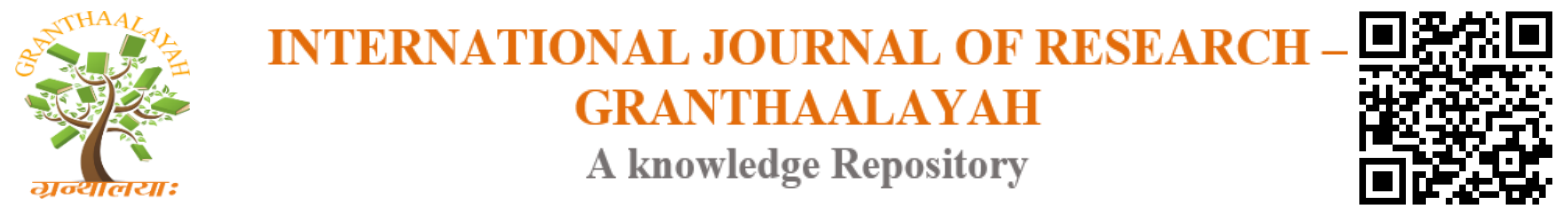

Science

\title{
CHARACTERIZATION OF PORTAL HYPERTENSION IN GEZIRASTATE (SUDAN) USING ULTRASOUND
}

\author{
Abdal Gadir Yonis ${ }^{1}$, Professor. Dr. Al Safi Ahmed Abdallash ${ }^{2}$, Dr.Mona Ahmed ${ }^{\text {, }}$, Dr. \\ Ashraf Mustafa Mohammed Osman 4 \\ ${ }^{1}$ AlshaabTeaching Hospital - Khartoum Sudan \\ ${ }^{2} \mathrm{MSc}, \mathrm{PhD}$, Professor of radiology in college of medical radiological sciences, Sudan University \\ ${ }^{3} \mathrm{MSc}, \mathrm{PhD}$, Associate Professor in Diagnostic Radiology in college of medical radiological \\ sciences, Sudan University -Sudan \\ ${ }^{4}$ MBBSc, MSc, PhD, Consultant Sonologist in Sahiroon specialized Hospital\& Al Zaitoona \\ Specialized Hospital
}

\begin{abstract}
Background

Peri-sinusoidal portal hypertension with frequent episodes of upper gastro intestinal variceal bleeding are hallmarks of hepatic-splenic schistosomiasis (Mansoni or Japonicum). This a prospective study compromise of 306 consecutive patients at North Gezira State between 2016 to 2019, aimed to characterize the portal hypertension \&to minimize potentiallysevere and deadly complications. Using B-mode grayscale ultrasound, The images done by portable Sono- ScapeA6 machine using convex probe with frequency range from 3.5 to $5 \mathrm{MHz}$ and the data analyzed by SPSS version 15.The result showed that the majority of patients were male and portal hypertension had high incidence associated with peri portal fibrosis(schistosomiasis), the result also showed that : out of 360 patients under study, there were 209(58.1\%) had portal hypertension, 106(29.4\%) had PPF, and 45(12.5\%) were normal patients. The study concluded that the portable ultrasound machine is the most frequently used imaging modality to assess patients for the presence of portal hypertension especially in the endemic area.
\end{abstract}

Keywords: Portal hypertension; B-Mode ultrasound; Schistomiasis and Portalvein.

Cite This Article: Abdal Gadir Yonis, Professor. Dr. Al Safi Ahmed Abdallash, Dr.Mona Ahmed, and Dr. Ashraf Mustafa Mohammed Osman. (2019). "CHARACTERIZATION OF PORTAL HYPERTENSION IN GEZIRASTATE $\quad$ (SUDAN) USING ULTRASOUND."International Journal of Research - Granthaalayah, 7(7), 30-38.

\section{Introduction}

The liver is a large organ in the body that cleans the blood and produces bile which helps the body to deal with the fats we eat. The portal venous system is the main vascular system supplying, the liver and other organs. Normal total liver blood flow is approximately $1600 \mathrm{ml} / \mathrm{minute} ; 1200 \mathrm{ml}$ 
(75\%) is delivered by the portal vein and $400 \mathrm{ml}$ by the hepatic artery. The liver receives $25-30 \%$ of the cardiac output. Blood flow through the liver is higher than in any other organ and it is understandable that changes in hepatic resistance may have marked circulatory consequences. Dooley J et al 1993.

The portal system includes all veins that carry blood from the abdominal part of the intestinal tract, the gall bladder, pancreas and spleen. Groszmann RJ et al 1994

Portal hypertension is a term used to describe elevated pressures in the portal venous system (a major vein that leads to the liver). Portal hypertension may be caused by intrinsic liver disease, obstruction, or structural changes that result in increased portal venous flow or increased hepatic resistance. Normally, vascular channels are smooth, but liver cirrhosis can cause them to become irregular and tortuous with accompanying increased resistance to flow.

This resistance causes increased pressure, resulting in avarices or dilations of the veins and tributaries. Pressure within the portal system is dependent upon both input from blood flow in the portal vein, and hepatic resistance to outflow. Normally, portal vein pressure ranges between 1-4 $\mathrm{mm} \mathrm{Hg}$ higher than the hepatic vein free pressure, and not more than $6 \mathrm{~mm} \mathrm{Hg} \mathrm{higher} \mathrm{than} \mathrm{right}$ atrial pressure. Pressures that exceed these limits define portal hypertension Groszmann RJ et al 1994.

Portal hypertension (PHT) is a pathologic increase in hydrostatic pressure in the portal venous system. Ninety percent of patients with PHT have pre sinusoidal or sinusoidal or post sinusoidal PHT.

Portal hypertension is mainly caused by liver cirrhosis, such as post hepatitis B or C or liver fibrosis such as schistosomiasis. The portal hypertension late stage problems include splenomegally, ascites and collaterals in the porto-systemic circulation. These will precipitate esophagealvericies can lead to upper gastrointestinal variceal bleeding and to death. The significant and the goal of the study will help in limiting the severity of portal hypertension to avoid the complication by detecting it in early stage as well as to decreased the morbidity and mortality and the risk factors, by characterized and assessed the liver and spleen morphology as well as the parameter in portal and splenic vein in the hepatic-splenic schistosomiasis choosing B-mode Gray scale ultrasound because its non-invasive methods and simple technique. Present studies were carried out by retrospectively reviewing the gray scale and Doppler ultrasound finding to evaluate its use fullness in patients of portal hypertension. Clinically, PHT is suspected in patients with splenomegaly and ascites, and the diagnosis is confirmed when Porto-systemic collateral channels are present (JiKai Yin 2007),

In cirrhosis the increase in portal pressure is clearly associated with an increase in outflow resistance and, indeed, portal blood flow and flow velocity tend to decrease as the disease gets worse and portal pressure builds up. (Fehr HF et al1993 \&Barbara L et al 1994)

Hepatosplenic schistosomiasis is a unique form of chronic fibrosing liver disease characterized by significant portal fibrosis with a preserved lobular parenchyma and a presinusoidal inflow block. 
Ultrasonography (US) is a non-invasive and inexpensive technique frequently used as first line examination in the diagnosis and follow-up of chronic hepatic diseases.US findings are usually highly specific, and can be considered sufficient to confirm the diagnosis G.H. Guyatt, et al 1991.

Ultrasound is the most frequently used imaging modality to assess patients for the presence of portal hypertension. Richard allan et al 2006.

Recent developments in digital technology have introduced various imaging modes, color/power Doppler, harmonic imaging for contrast enhancement, three-dimensional visualization, and fusion imaging. (BaikSK et al 2014 \& Baik 2010 and Yokosuka O, et al 2016) However, fundamental tissue images are available only using B-mode sonography. The role of this simple technique for portal hypertension is to characterize cirrhosis, preiportal fibrosis measure vessel diameter and spleen size, and identify the ascites and abnormal collateral route. (Pilette C, et al. 1997 \& Yokosuka O et al 2015).

\section{Material and Methods}

This is a prospective study aimed to characterize the portal hypertension used B-mode grayscale ultrasound, compromise of 360,45 patient have normal liver, 209 patients have portal hypertension, and 106 patients have peri portal fibrosis .carried out in the period from January 2016 to 2019 in north Gazira state in Sudan and private clinic at Gebel Awlia city, Khartoum state requested abdominal US was done with prior explanation of the investigation and informed written consent of the patients .ultrasound done was performed on SonoScape- A6 portable machine using convex probe with frequency range from 3.5 to $5 \mathrm{MHz}$ liver was scanned in various planes like transverse, sagittal, sub costal ,obliges inter costal and coronal planes. Comparative upper abdominal organs were done.

Various ultra-sonographic features were observed, which included overall assessment of liver size, portal vein, gall bladder wall thickness, splenic size, splenic vein, collateral, and ascites. These features was collected in a collection sheet(questionnaire), all questionnaires were daily checked from completeness and accuracy by investigator .filled questionnaires were coded before entering data into computer using statistical package for social science(SPSS) version 15. Statistical analysis was performed using SPSS version 15.

\section{Result}

This is a three years prospective study of characterization of portal hypertension using gray scale B-mode ultrasound in 360 patients at Gazira state and private clinic at Gebel Awlia, Khartoum state. The study showed in tables and figures as follows:

Table 1: Shows Gender distribution

\begin{tabular}{|l|l|l|l|}
\hline \multicolumn{2}{|c|}{} & Frequency & Percent \\
\hline Valid & Male & 244 & $67.8 \%$ \\
\cline { 2 - 4 } & Female & 116 & $32.2 \%$ \\
\cline { 2 - 4 } & Total & 360 & $100.0 \%$ \\
\hline
\end{tabular}


Table 2: showed the ultrasound finding

\begin{tabular}{|l|l|l|l|}
\hline \multicolumn{2}{|c|}{} & Frequency & Percent \\
\hline \multirow{3}{*}{ Valid } & Normal & 45 & 12.5 \\
\cline { 2 - 4 } & Portal Hypertension & 209 & 58.1 \\
\cline { 2 - 4 } & PPF & 106 & 29.4 \\
\hline & Total & 360 & 100.0 \\
\hline
\end{tabular}

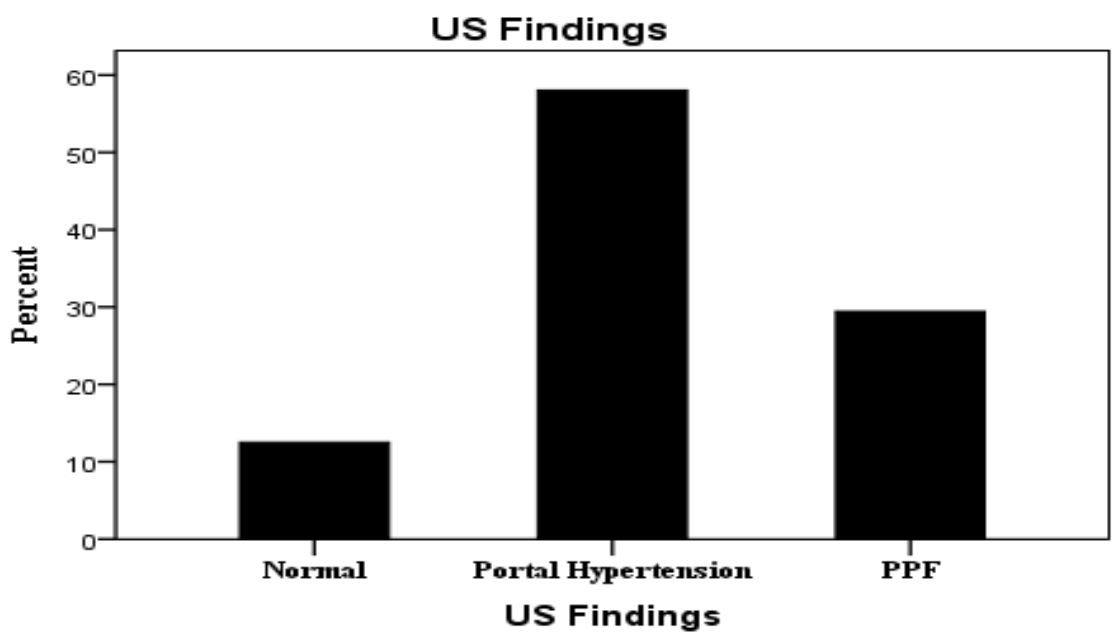

Figure 1: below, a 2-D Bar chart show the ultrasound finding

Table 3: of distribution of Causes

\begin{tabular}{|c|c|c|}
\hline & Frequency & Percent \\
\hline Normal & 45 & 12.5 \\
\hline Schistomasis & $(198 \mathrm{ph}+105 \mathrm{ppf}) 304$ & 84.4 \\
\hline Alcoholic Cirrhosis & & 2.3 \\
\hline Viral Cirrhosis & 3 & .8 \\
\hline Total & 360 & 100.0 \\
\hline
\end{tabular}

Table 4: cross-tabulation table show the relation between mean of PV Width $(\mathrm{cm})$ SV Width $(\mathrm{Cm})$ Spleen size $(\mathrm{Cm})$ and group statistics control and Patients group.

Group Statistics

\begin{tabular}{|l|l|l|l|l|l|}
\hline & TYPE & N & Mean & Std. Deviation & Std. Error Mean \\
\hline PV Width (Cm) & Control & $\mathbf{4 5}$ & $\mathbf{1 . 0 3 2 2}$ & $\mathbf{1 3 3 5 7}$ & .01991 \\
\cline { 2 - 5 } & Patient Group & $\mathbf{3 1 5}$ & $\mathbf{1 . 4 3 0 3}$ & $\mathbf{1 8 3 8 6}$ & $\mathbf{0 1 0 3 6}$ \\
\hline \multirow{2}{*}{ SV Width (Cm) } & Control & $\mathbf{4 5}$ & $\mathbf{. 6 3 0 7}$ & $\mathbf{. 1 1 5 5 5}$ & $\mathbf{0 1 7 2 3}$ \\
\cline { 2 - 6 } & Patient Group & $\mathbf{3 1 5}$ & $\mathbf{1 . 0 4 4 3}$ & $\mathbf{. 1 4 3 6 8}$ & $\mathbf{0 0 8 1 0}$ \\
\hline \multirow{2}{*}{ Spleen size (Cm) } & Control & $\mathbf{4 5}$ & $\mathbf{1 0 . 0 1 7 8 . 7 5 2 6 6}$ & $\mathbf{. 1 1 2 2 0}$ \\
\cline { 2 - 5 } & Patient Group 315 & $\mathbf{1 5 . 7 9 2 1} 2.50673$ & $\mathbf{. 1 4 1 2 4}$ \\
\hline
\end{tabular}




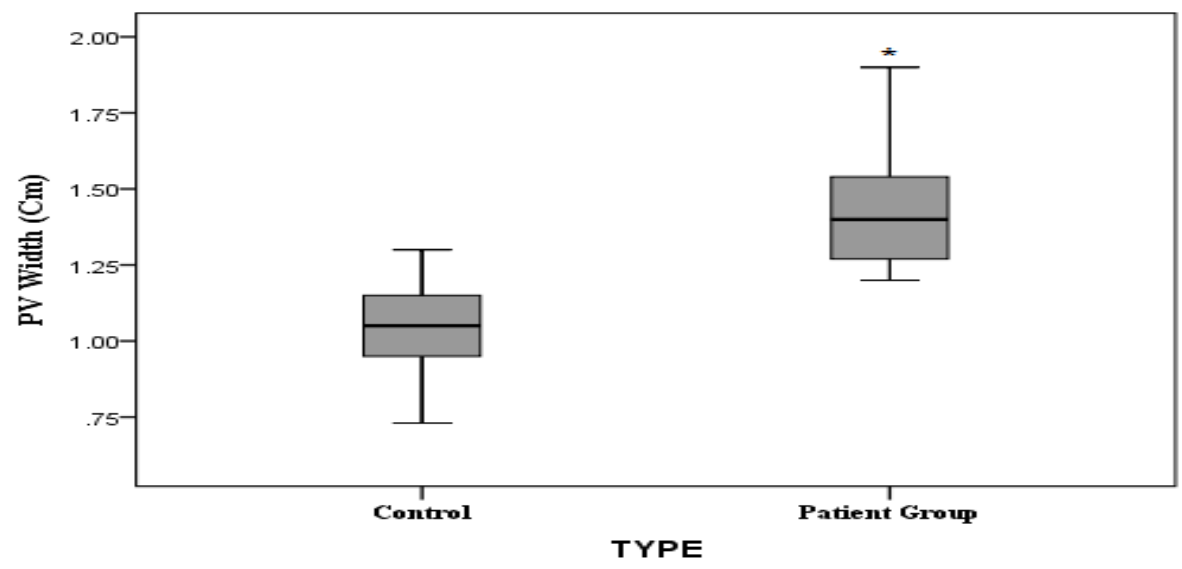

Figure 2: mean of PV Width in control and Patients group. The strike indicates significant difference $(P<0.05)$.

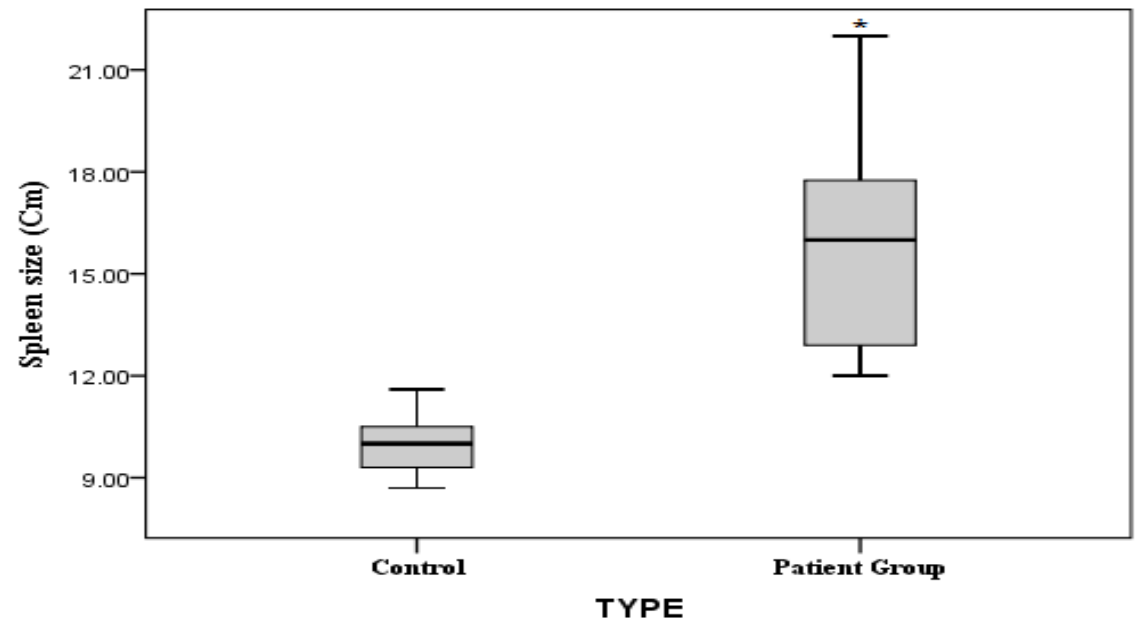

Figure 3: mean of spleen size in control and Patients group. The strike indicates significant difference $(P<0.05)$.

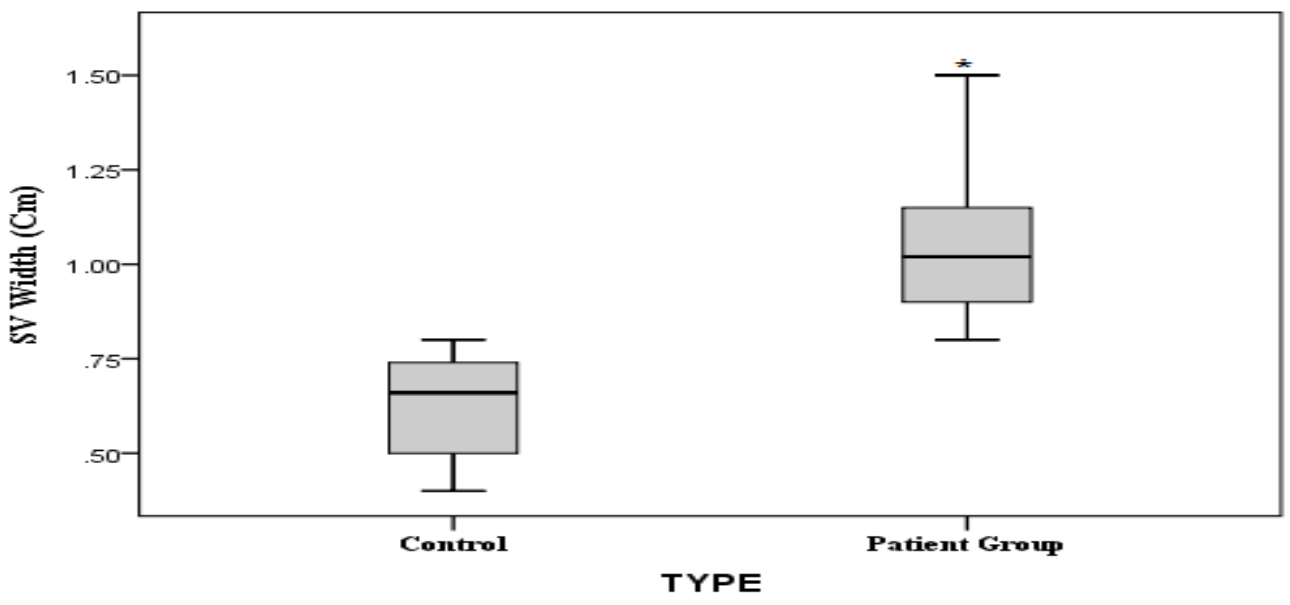


Table 5: cross-tabulation table show the relation between of the mean PV Width $(\mathrm{cm})$ SV Width (cm) Spleen size $(\mathrm{cm})$ and group control normal and Patients group PPF and PHT.

\begin{tabular}{|l|l|l|l|}
\hline & & N & Mean \\
\hline PV Width (Cm) & Normal & 45 & 1.03 \\
\cline { 2 - 4 } & PPF & 106 & 1.25 \\
\cline { 2 - 4 } & Portal Hypertension 209 & 1.52 \\
\hline SV Width (Cm) & Normal & 45 & .63 \\
\cline { 2 - 4 } & PPF & 106.887 \\
\cline { 2 - 4 } & Portal Hypertension 209 & 1.12 \\
\hline Spleen size (Cm) & Normal & 45 & 10.02 \\
\cline { 2 - 4 } & & 106 & 12.68 \\
\cline { 2 - 4 } & PPF & Portal Hypertension 209 & 17.37 \\
\cline { 2 - 4 } & &
\end{tabular}

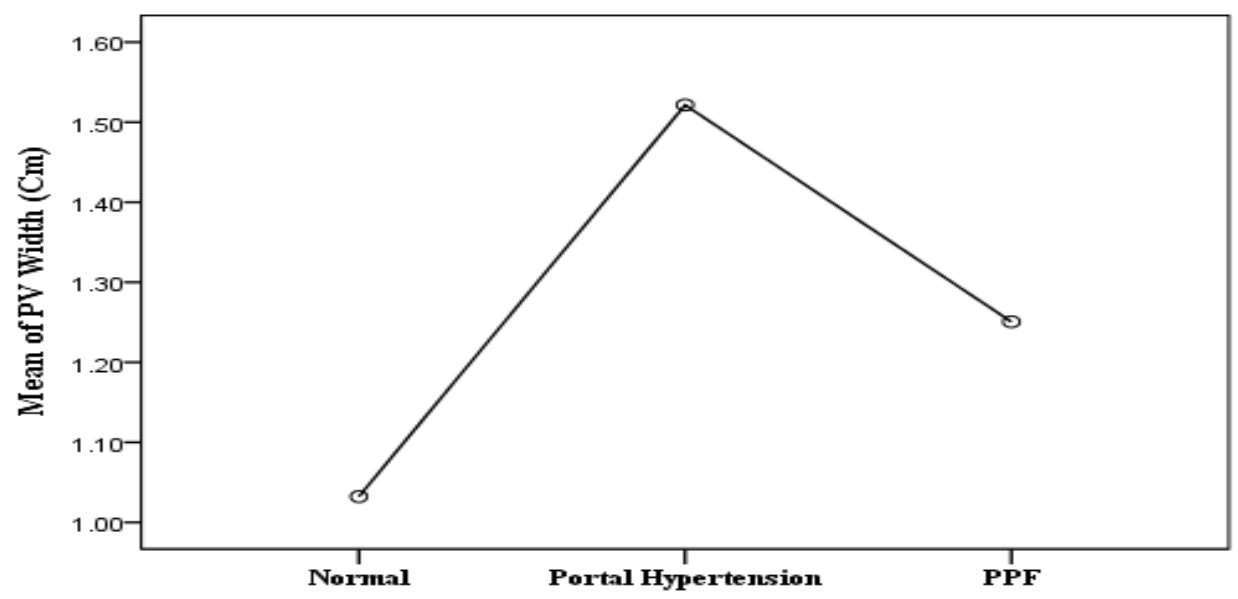

Figure 4: The main portal vein diameter in a 2-D line chart show the distribution of PPF \&P HTN patents related to normal.

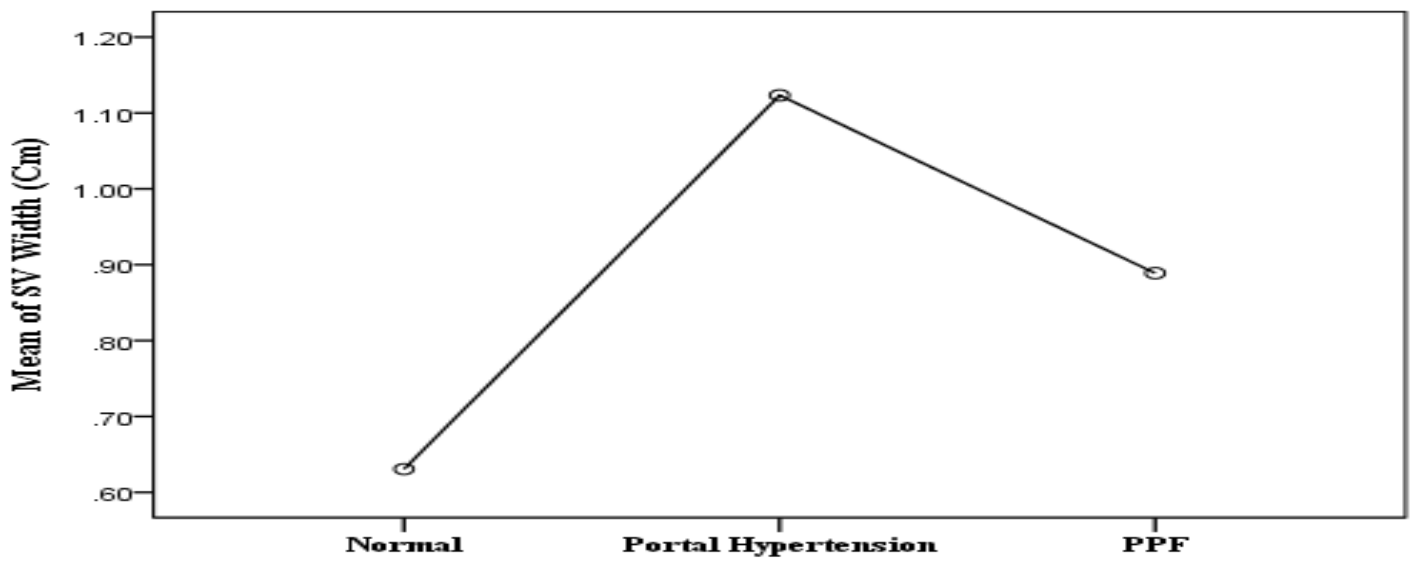

Figure 5: The splenic vein diameter in a 2-D line chart show the distribution of PPF \& P HTN patents related to normal. 


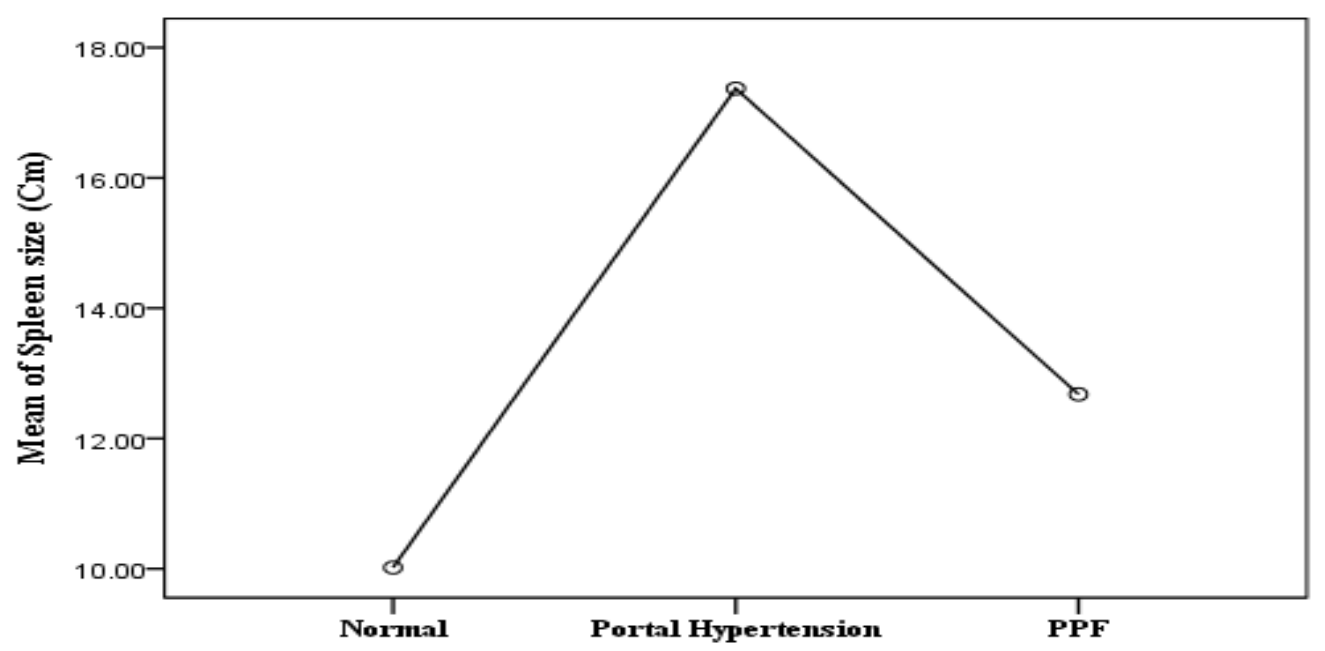

Figure 6: The splenic size (span) in a 2-D line chart show the distribution of PPF \& P HTN patents related to normal.

\section{Discussion}

There were 360 patients, 244(67.8\%) males and $116(32.2 \%)$ females with an age range of 30 to 72 years (mean) were studied (table 1), the study showed that :out of 360 patients under study, there were 209(58.1\%) had portal hypertension,106(29.4\%) had PPF, and 45(12.5\%) were normal patients. There were highly incidence of portal hypertension patients due to the majority of patient had schistosomiasis advance stage state showed that table (1) and figure (1).

According to the ultrasound finding of the diseases and the causes, the study showed the majority of the patients had periportal fibrosis with portal hypertension due to schistosomiasisin 55\% of cases, had periportal fibrosis without portal hypotension due to schistosomiasis in $29.4 \%$, and had portal hypertension due to liver cirrhosis in 3.1\% (alcoholic $2.3 \%$ and viral cirrhosis .8\%). (Table2\& 3) this mean that the schistosomiasismansoni in the endemic area is play a key role in causes of periportal fibrosis and PHT. This was in accordance with those previous studies which were recorded the result of PHT and PPF like whom Burchard et al.1998, concluded that PPF mainly due to schistosomiasis. In this study alcoholic is not responsible for fibrosis, chronic hepatitis does not play any role in the population of the study area, as causes chronic liver diseases. Table (3)

Of this study sonographic visualization of the portal vessels was obtained in most cases in patient with schistosomiasis was significantly greater when compare with the mean obtained for in individual, and in the normal control group. The increase in the width of the splenic vein was impressive, its mean that the width in patient with PHT was twice the width measured in the control group because the splenic vein don't effected of collateral while the portal vein lack of the sensitivity due to collateral path way which partially decompress the system . The upper limit of the normality for the mean width of portal was set through $1.03 \mathrm{~cm}$ (Std. Deviation 0.14) for the portal vein $0.63 \mathrm{~cm}$ (Std. Deviation 0.12) for the splenic vein width. The mean width over $1.43 \mathrm{~cm}($ Std. Deviation 0.18 ) in group patient (periportal fibrosis the mean width $1.25 \mathrm{~cm}$ and $1.52 \mathrm{~cm}$ for portal hypertension) for the portal vein, and $1.04 \mathrm{~cm}(S t d$. Deviation 0.15$)$ for the 
splenic vein in group patient (in periportal fibrosis $0.89 \mathrm{~cm}+$ in portal hypertension $1.12 \mathrm{~cm}$ ) were $\% 100$ specific for the diagnosis of portal hypertension.

The upper limit of normality for the width of the portal vessels has not yet firmest established for the portal vein, for example some others have chosen $12 \mathrm{~mm}$ while other suggested $20 \mathrm{~mm}$. Indicated that the best discriminated vessels for the diagnosis of PHT this agree with all pervious study in any research which were recorded result of the spleen vein was(sensitivity of \%85 and specifically\%96 followed by the portal vein sensitivity $\% 76$ and specifically of \%90) it was noting that Zoli et al The upper limit of the normality for the mean width of spleen size was set through $10.02 \mathrm{~cm}(\mathrm{Std}$. Deviation 0.75$)$.In group patient The mean width of spleen size over $17.79 \mathrm{~cm}(\mathrm{Std}$. Deviation 2.51) (in periportal fibrosis $12.68 \mathrm{~cm}+$ in portal hypertension $17.37 \mathrm{~cm}$ ) in this study the strike indicate significant difference $(P<0.05)(0.014)$, on other hand the enlarged spleen is of low significant according to deferent diseases, like acute involvement of the spleen such as in malaria and anemia. Our study agrees with. Bru, J. Bosch et al spleen dimension is the US sing most commonly associated to the presence of PHT contrarily to other sing \&it is sensitivity is high, while is ranges 50-60\% according to different series it's an independent predictor of esophageal varies(Table4)\&Figures (2,3and4). There were $45(12.5 \%)$ cases control group out of 360 cases under study The upper limit of the normality for the mean width of portal was set through $1.03 \mathrm{~cm}$ for the portal vein $0.63 \mathrm{~cm}$ for the splenic vein width and $10.02 \mathrm{~cm}$ for the spleen size in 106 cases The mean width over $1.25 \mathrm{~cm}$ for the portal vein, and $0.89 \mathrm{~cm}$ for the splenic vein and $12.68 \mathrm{~cm}$ for spleen size in group patient (periportal fibrosis without portal hypertension) were 209 cases the mean $17.37 \mathrm{~cm}$ for spleen size in patient with $\% 100$ specific for the diagnosis of portal hypertension. In figure( $)$ The strike indicate significant difference $(P<0.05)$ There were 45 $(12.5 \%)$ cases out of 360 cases under study have normal PV width, 106 (29.4\%) patients have mild dilatation, $170(47.2 \%)$ patients have moderate dilatation and $39(10.8 \%)$ patients have severe dilatation. Table (5) plus figures (5, 6 and 7).

\section{Conclusion}

Ultrasonography (US) is a non-invasive and inexpensive technique frequently used as first line examination in the diagnosis and follow-up of chronic hepatic diseases.US findings are usually highly specific, and can be considered sufficient to confirm the diagnosis. The portable Ultrasound machine is the most frequently used imaging modality to assess patients for the presence of portal hypertension due to schistosomiasis especially in the endemic area.

\section{References}

[1] Hemodynamic Features of Doppler Ultrasonography in Patients With Portal Hypertension. Intraoperative Direct Measurement of Portal Pressure in the Portal Venous System Li Zhang, PhD, Yun-You Duan, PhD, Jin-Mao Li, MD, Ji-Kai Yin, PhD 2007 by the American Institute of Ultrasound in Medicine • J Ultrasound Med 2007; 26:1689-1696•0278-4297/07/\$3.50.

[2] Baik SK. Haemodynamic evaluation by Doppler ultrasonography in patients with portal hypertension: a review. Liver Int.2010;30:1403-1413Gaiani S, Bolondi L, Li Bassi S, ZironiG, Siringo S, Barbara L.

[3] Prevalence of spontaneous hepatofugal flow in livercirrhosis. Gastroenterology. 1991;100:1607.ultrasound assessment of portal hypertension by richardallan, ams, head sonographer, flinders medical centre, sa.22 issue 1, 2006 
[4] Kim MY, Jeong WK, Baik SK. Invasive and non-invasive diagnosisof cirrhosis and portal hypertension. World J Gastroenterol 2014;20:4300-4315.

[5] Oberti F, Valsesia E, Pilette C, et al. Noninvasive diagnosis of hepatic fibrosis or cirrhosis. Gastroenterology 1997;113:1609-1616.

[6] Maruyama H, Kondo T, Sekimoto T, Yokosuka O. Differential clinical impact of ascites in cirrhosis and idiopathic portal hypertension. Medicine (Baltimore) 2015;94:e1056.

[7] Genecin P, Groszmann RJ. The biology of portal hypertension. In: Arias IM, BoyerJL, Fausto N, Jakoby WB, Schachter DA, Shafritz DA, editors. The Liver: Biology and Pathobiology. New York: Raven press, Ltd.; 1994. p. 1327-1341.G.-D.

[8] Burchard et al.1998 Ultrasound and chemical markers of hepatosplenicschistosomiasis.

[9] Maruyama H, Shiha G, Yokosuka O, et al. Non-invasive assessment of portal hypertension and liver fibrosis using contrast enhanced ultrasonography. HepatolInt 2016;10:267-276.

[10] Schmassmann A, Zuber M, Livers M, Jager K, Jenzer HR, Fehr HF. Recurrent bleeding after variceal hemorrhage: predictive value of portal venous duplex sonography. AJR Am J Roentgenol. $1993 ; 160: 41-7$

[11] Siringo S, Bolondi L, Gaiani S, Sofia S, Di Febo G, Zironi G, Rigamonti A, Miglioli M, Cavalli G, Barbara L. The relationship of endoscopy, portal Doppler ultrasound flowmetry, and clinical and biochemical tests in cirrhosis.JHepatol.1994;20:11-8

[12] Sherlock S, Dooley J. Diseases of the liver and biliary system. Ninth ed. Oxford: Blackwell Scientific Publications; 1993.

\footnotetext{
*Corresponding author.

E-mail address: ashrafmerafabi@ gmail.com
} 